\title{
Correction to: Mitochondrial dynamics regulators: implications for therapeutic intervention in cancer
}

\author{
Sanjay Kumar ${ }^{(D)}$ Rahail Ashraf • Aparna C.K.
}

Published online: 4 March 2022

(C) Springer Nature B.V. 2022

\section{Correction to: Cell Biol Toxicol https://doi.org/10.1007/s10565-021-09662-5}

Unfortunately, the original version of this article contained a mistakes.

In the abstract section, "mitochondWangrial dynamics" should be corrected as mitochondrial dynamics.

In figure legends $2 \& 3$ ) an image before =inhibition is missing. The corrected sentence in Figs. 2 and 3 legends are shown below.

In Fig. 2 legend, the sentence (=inhibition, $\mathrm{CSCs}=$ cancer stem cells, EMT $=$ epithelial to mesenchymal transition, BTIC $=$ Brain tumor initiating cells) should be corrected as ( $\perp=$ inhibition, $\mathrm{CSCs}=$ cancer stem cells, EMT $=$ epithelial to mesenchymal transition, BTIC $=$ Brain tumor initiating cells).

The original article can be found online at https://doi.org/ 10.1007/s10565-021-09662-5.

S. Kumar $(\bowtie) \cdot$ R. Ashraf · A. C.K.

Division of Biology, Indian Institute of Science

Education and Research (IISER) Tirupati, Rami

Reddy Nagar, Karkambadi Road, Mangalam, Tirupati,

Andhra Pradesh 517507, India

e-mail: sanjay@iisertirupati.ac.in; sanjay28@gmail.com
In Fig. 3 legend, the sentence MYLS22 inhibits OPA1 and reduces tumor growth, angiogenesis, and metastasis (Herkenne et al. 2020) (=inhibition) should be corrected as MYLS22 inhibits OPA1 and reduces tumor growth, angiogenesis, and metastasis (Herkenne et al. 2020) ( $\perp=$ inhibition).

The original article has been corrected.

Publisher's note Springer Nature remains neutral with regard to jurisdictional claims in published maps and institutional affiliations. 